\title{
Synthesis and Electrophysical Properties of Ultrahigh-Molecular-Weight Polyethylene and Carbon Nanotubes Based Composites
}

\author{
Ilya A. Markevicha, Gennady E. Selyutin ${ }^{\mathrm{b}}$, \\ Nikolai A. Drokin*c and Boris A. Belyaev \\ ${ }^{a}$ Siberian Federal University \\ 79 Svobodny, Krasnoyarsk, 660041, Russia \\ ${ }^{b}$ Institute of Chemistry and Chemical Technology SB RAS \\ FRC "Krasnoyarsk Science Center SB RAS" \\ 50/24 Akademgorodok, Krasnoyarsk, 660036, Russia \\ ${ }^{c}$ L.V. Kirensky Institute of Physics SB RAS \\ FRC "Krasnoyarsk Science Center SB RAS" \\ 50/38 Akademgorodok, Krasnoyarsk, 660036, Russia
}

Composites based on ultrahigh-molecular-weight polyethylene and carbon nanotubes were obtained by mixing the components in xylene at certain temperatures. The complex conductivities and permittivities of the material samples were measured in the frequency range from $100 \mathrm{~Hz}$ to $1 \mathrm{GHz}$. The conditions for the synthesis of both electrically conducting and dielectric composites were established. The conductivity depends not only on the concentration of carbon nanotubes, but also on the structure of their distribution in the polymer matrix, which in turn depends on the temperature conditions for obtaining the composite.

Keywords: composites, ultrahigh-molecular-weight polyethylene, carbon nanotubes, dielectric permittivity, conductivity.

Citation: Markevich I.A., Selyutin G.E., Drokin N.A., Belyaev B.A. Synthesis and electrophysical properties of ultrahighmolecular-weight polyethylene and carbon nanotubes based composites, J. Sib. Fed. Univ. Eng. technol., 2018, 11(2), 190-197. DOI: $10.17516 / 1999-494 X-0022$.

(c) Siberian Federal University. All rights reserved

* Corresponding author E-mail address: 4ubekpam@mail.ru, drokin@iph.krasn.ru 


\title{
Синтез и электрофизические свойства композитов на основе сверхвысокомолекулярного полиэтилена и углеродных нанотрубок
}

\author{
И.А. Маркевич ${ }^{a}$, Г.Е. Селютин ${ }^{\sigma}$, \\ Н.А. Дрокин ${ }^{\text {, Б.А. Беляев }}{ }^{\text {B }}$ \\ ${ }^{a}$ Сибирский федеральный университет \\ Россия, 660041, Красноярск, пр. Свободный, 79 \\ ${ }^{6}$ Институт химии и химической технологии СО РАН \\ ФИЦ «Красноярский научный иентр СО РАН» \\ Россия, 660036, Красноярск, Академгородок, 50/24 \\ ${ }^{6}$ Институт физики им. Л.В. Киренского СО РАН \\ ФИЦ «Красноярский научный центр СО РАН» \\ Россия, 660036, Красноярск, Академгородок, 50/38
}

Получены композиты на основе сверхвысокомолекулярного полиэтилена и углеродных нанотрубок методом смешивания компонентов в ксилоле при определенных температурах. Комплексные проводимости и дилектрические проницаемостиобразиовматериалов измерень в диапазоне частот от 100 Ги до 1 ГГи. Установлены условия синтеза как электропроводящих, так и диэлектрических композитов. При этом величина проводимости зависит не только от концентрации углеродных нанотрубок, но и от структуры их распределения в полимерной матрице, которая, в свою очередь, зависит от температурных условий получения композита.

Ключевые слова: композиты, сверхвысокомолекулярный полиэтилен, углеродные нанотрубки, диэлектрическая пронииаемость, проводимость.

\section{Введение}

В настоящее время в научной литературе растет число работ, посвященных изучению композиционных материалов на основе сверхвысокомолекулярного полиэтилена (СВМПЭ), модифицированного углеродными нанотрубками (УНТ) [1]. Это связано с тем, что такие материалы имеют большую перспективу применения в самых разных областях техники и технологии. Действительно, СВМПЭ обладает уникальным набором физических свойств: высокой износостойкостью, стойкостью к ударным нагрузкам и агрессивным средам, широким температурным диапазоном эксплуатации (от $100{ }^{\circ} \mathrm{C}$ до криогенных температур). Введение в СВМПЭ углеродных нанотрубок до определенных концентраций не только повышает физико-механические характеристики полимера [2], но и переводит его в класс электропроводящих материалов [3]. В зависимости от своих электрических свойств (диэлектрик либо проводник) композит весьма перспективен для использования в радиоэлектронике и СВЧтехнике в качестве радиопрозрачных или экранирующих материалов, надежно защищающих при этом аппаратуру от климатических и внешних механических воздействий (ударов, агрессивных сред).

Концентрация проводящего наполнителя является основным параметром, определяющим электрические свойства полимерного композита. Однако помимо этого на электрические ха- 
рактеристики материала также оказывает влияние однородность распределения проводящих включений в объеме матрицы, которая зависит от метода и условий приготовления композита. Существуют различные способы получения полимерных композитов с нанотрубками: перемешивание соответствующих компонентов в мешалках, планетарных мельницах, ступках, смешивание в различных растворителях или в расплаве полимера и in-situ полимеризация [4, 5]. Метод смешивания в растворителе наиболее универсальный из них, так как позволяет приготавливать композиты с различной однородностью распределения наполнителя [6] за счет гибкой регулировки параметров метода. Исследования по полученным по этой методике композитам, описанные во множестве научных публикаций, представлены в обзоре [1]. Эти композиты имеют разнообразные электрические свойства, так как отличаются типом полимера, типом и концентрацией нанотрубок, параметрами метода получения, способом изготовления образцов. При этом не просматриваются закономерности, какой из множества факторов оказывает решающее влияние на характеристики композита. Это затрудняет понимание того, каковы реальные возможности применения конкретного метода для синтеза материалов с заданными свойствами. В результате целью настоящей работы является определение возможности использования техники смешивания компонентов в растворителе для приготовления как электропроводящих, так и диэлектрических СВМПЭ-композитов, а также изучение их электрофизических характеристик.

\section{Методика получения композитов и методика измерений}

Для определения возможностей метода смешивания в расторителе композиты были синтезированы единым образом. Изменялась лишь температура смешивания компонентов. Использовался СВМПЭ компании Braskem (молекулярная масса 6.4 $10^{6}$ г/моль, средний размер частиц 150 мкм) и многостенные углеродные нанотрубки (МУНТ) различной морфологии с чистотой продукта 99 \%, со средним диаметром 7 нм, длиной 2-2.5 мкм и проводимостью 2500 См/м, полученные в Институте катализа СО РАН (г. Новосибирск).

Образцы композитов, содержащие 1 вес. \% МУНТ, получали по следующей методике. Порошок МУНТ в ксилоле обрабатывали ультразвуком интенсивностью 290 Вт/см ${ }^{2}$ в течение 30 мин до формирования однородной суспензии. В три приготовленные суспензии МУНТ с температурами 90 (образец № 1), 110 (образец № 2) и $144{ }^{\circ} \mathrm{C}$ (образец № 3) засыпали порошок СВМПЭ. Далее производилось перемешивание составляющих компонентов гомогенизатором MPW-309 при 1000 об/мин в течение 10 мин до визуально однородного состояния. Для образца № 3 из-за высокой температуры приготовления $144{ }^{\circ} \mathrm{C}$ (температура кипения ксилола), стадия перемешивания гомогенизатором была заменена ультразвуковой обработкой интенсивностью $290 \mathrm{BT} / \mathrm{cm}^{2}$ в течение 30 мин до формирования однородной смеси. После фильтрования и сушки смесей получали порошки композитов, из которых горячим прессованием при 6 МПа и $160{ }^{\circ} \mathrm{C}$ изготавливали экспериментальные образцы в виде дисков диаметром 16 мм и толщиной $\sim 1$ мм.

Микрофотографии композитов (рис. 1) были сделаны на оптическом микроскопе АХIO Imager.A1m.

Изучение комплексной проводимости и комплексной относительной диэлектрической проницаемости (ДП) синтезированных композитов проводили в диапазоне частот от 100 Гц до 


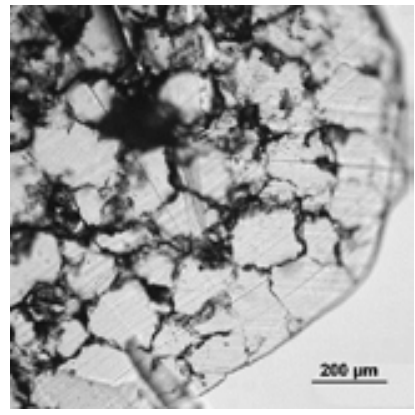

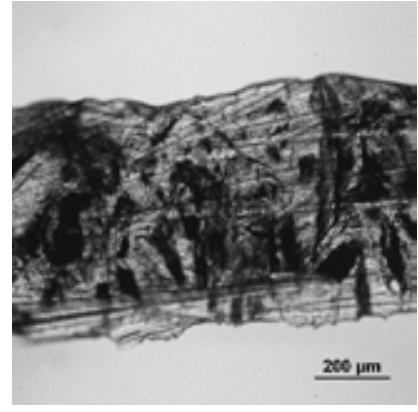

6

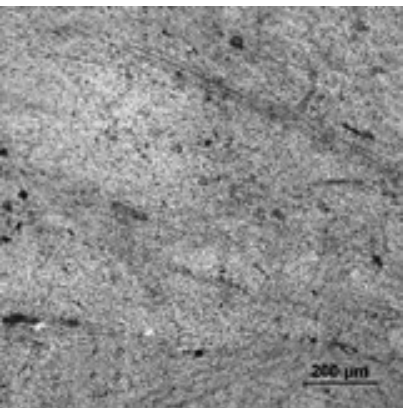

B

Рис. 1. Микрофотографии образцов композитов: а - образец № 1; б - образец № 2; в - образец № 3

Fig. 1. Photomicrography of composite samples: - sample No. 1; б - sample No. 2; в-sample No. 3

1 ГГц методом измерения импеданса с использованием векторного анализатора цепей Е5061B (Agilent Technology). Для проведения измерений исследуемый образец помещали между металлическими обкладками конденсатора, которые изготавливались в виде тонких слоев индия, прикатанных к торцевым поверхностям образца.

\section{Результаты и их обсуждение}

На рис. 2 в логарифмическом масштабе приведены частотные зависимости действительных и мнимых компонент удельной проводимости и диэлектрической проницаемости образца № 1 , рассчитанные из импедансных измерений.

Как видно на рис. $2 a$, активная компонента проводимости $\sigma^{\prime}$ практически постоянна $\left(\sigma^{\prime}=0.018 \mathrm{CM} / \mathrm{M}\right)$ в диапазоне частот от 100 Гц до 1 МГц, и лишь на частотах $f>10^{7}$ Гц она начинает расти, достигая значения $\sigma^{\prime}=0.15 \mathrm{Cm} / \mathrm{M}$. Реактивная (емкостная) компонента проводимости $\sigma$ ” в логарифмических координатах линейно возрастает. Такое поведение проводимости объясняется дисперсией действительной и мнимой компонент ДП (рис. 2б).

Действительная компонента ДП $\varepsilon^{\prime}$ на частоте $10^{2}$ Гц соответствует величине $\varepsilon^{\prime}=87$ и плавно уменьшается до $\varepsilon^{\prime}=3.4$ в СВЧ-области. Это можно объяснить тем, что в композите большие значения действительной компоненты $\varepsilon$ в в области низких частот возникают в результате поляризации проводящих агломератов нанотрубок, разделённых диэлектрической прослойкой полимера (поляризация по механизму Максвелла-Вагнера) [7]. При увеличении частоты процессы поляризации сначала больших, затем меньших комплексов из МУНТ не успевают в силу инерции за действующим переменным электрическим полем, и диэлектрическая проницаемость постепенно уменьшается.

На рис. 3 изображены в логарифмическом масштабе частотные зависимости действительных и мнимых компонент удельной проводимости и диэлектрической проницаемости образца № 2 .

Низкочастотная или статическая проводимость композита № 2 на частоте 100 Гц равна $4.8 \cdot 10^{-6} \mathrm{Cm} / \mathrm{м}$ (рис. $3 a$ ). Она оказалась существенно меньше, чем низкочастотная проводимость образца № 1, и при увеличении частоты монотонно возрастает более чем на 4 порядка. Такое возрастание проводимости характерно для многих неоднородных по составу или структуре 


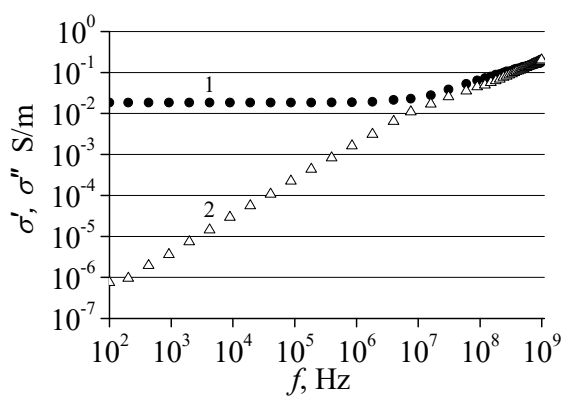

a

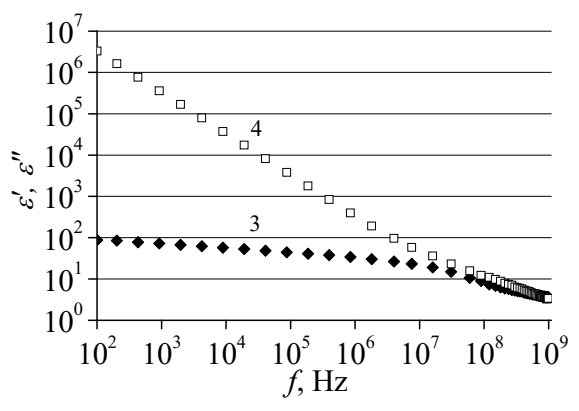

б

Рис. 2. Частотные зависимости комплексной проводимости (а) и комплексной диэлектрической проницаемости (б) образца № 1: $1-\sigma ; 2-\sigma^{\prime \prime} ; 3-\varepsilon^{\prime} ; 4-\varepsilon$,

Fig. 2. Frequency dependencies of the complex conductivity (a) and the complex dielectric permittivity (б) of the sample No. 1: $1-\sigma^{\prime} ; 2-\sigma^{\prime} ; 3-\varepsilon ; 4-\varepsilon$ '

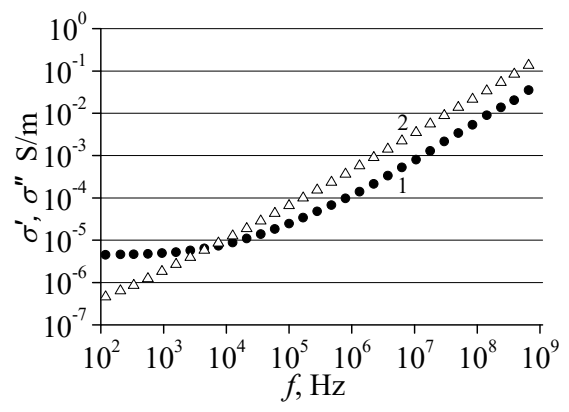

a

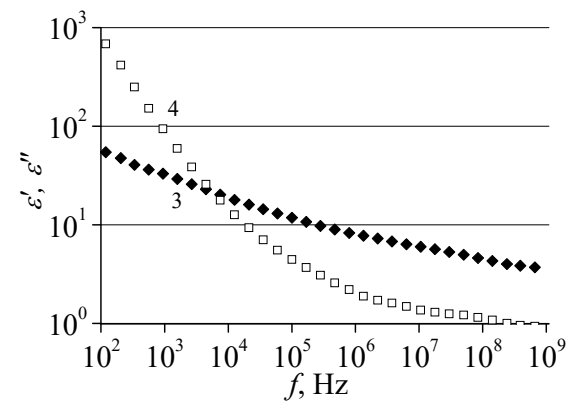

6

Рис. 3. Частотные зависимости комплексной проводимости (a) и комплексной диэлектрической проницаемости (б) образца № 2: $1-\sigma ; 2-\sigma^{\prime \prime} ; 3-\varepsilon^{\prime} ; 4-\varepsilon$ ”'

Fig. 3. Frequency dependencies of the complex conductivity (a) and the complex dielectric permittivity (б) of the sample No. 2: $1-\sigma ; 2-\sigma ' ; 3-\varepsilon ; 4-\varepsilon "$

материалов, обладающих совокупностью термоактивационной, туннельной и прыжковой проводимостей [8].

Дисперсия $\varepsilon^{\prime}(f)$ образца № 2 наблюдается во всем частотном диапазоне 100-109 Гц (рис. 3б). Действительная компонента ДП снижается от величины $\varepsilon^{\prime}=57$ на частоте 100 Гц до значения $\varepsilon^{\prime}=3.6$ при $10^{9}$ Гц. Как и в образце № 1 , мнимая компонента ДП $\varepsilon$ ”' также возрастает с понижением частоты за счет вклада от активной проводимости.

На рис. 4 приведены результаты измерений электрофизических характеристик образца № 3.

Активная и реактивная компоненты проводимости данного материала с ростом частоты пропорционально увеличиваются. На частоте 100 Гц действительная часть проводимости образца № 3 на шесть порядков ниже, чем в образце № 1, и на два порядка - чем в № 2. Возрастание реальной компоненты проводимости $\sigma$ ' в широком диапазоне частот можно объяснить преодолением носителями зарядов различных по величине потенциальных барьеров в композите или путём туннелирования в системе беспорядочно расположенных нанотрубок. Такая про- 


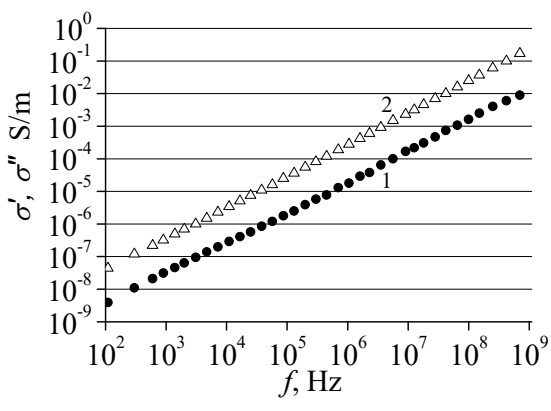

a

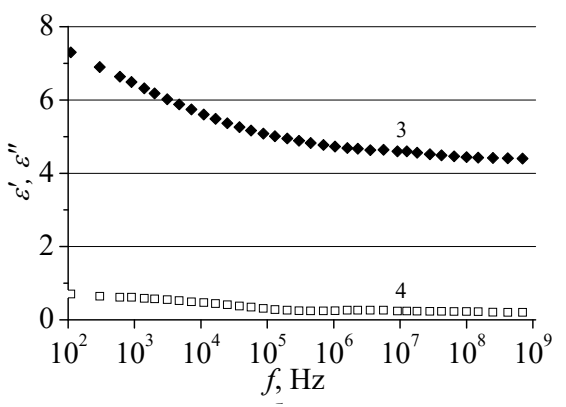

6

Рис. 4. Частотные зависимости комплексной проводимости $(a)$ и комплексной диэлектрической проницаемости (б) образца № 3: $1-\sigma ; 2-\sigma^{\prime \prime} ; 3-\varepsilon ; 44-\varepsilon$ ’’

Fig. 4. Frequency dependencies of the complex conductivity (a) and the complex dielectric permittivity (б) of the sample No. 3: $1-\sigma ' ; 2-\sigma ' ; 3-\varepsilon ; 44-\varepsilon$,

водимость может возникать в условиях, когда нанотрубки случайно распределяются в объёме материала и отсутствует непосредственный контакт между ними. В этом случае статическая проводимость будет мала и в данном образце не превышает значений $\sigma^{\prime}=4 \cdot 10^{-8} \mathrm{Cm} / \mathrm{m}$. Наличие такой системы изолированных друг от друга нанотрубок в веществе косвенно подтверждается и характером частотной зависимости реальной компоненты диэлектрической проницаемости, которая представлена на рис. 46 в линейном масштабе. Видно, что обе компоненты ДП монотонно уменьшаются с ростом частоты. Однако зависимость ДП от частоты не такая яркая, как в образцах № 1 и № 2. Следует отметить, что диэлектрическая проницаемость данного материала возросла примерно в 2-3 раза по сравнению с чистым СВМПЭ $\left(\varepsilon^{\prime}=2.5\right)$.

Поведение электрофизических характеристик композитов объясняется специфическим распределением нанотрубок в матрице СВМПЭ, которое зависит от условий приготовления материала. Структура распределения нанотрубок в полимере хорошо видна на микрофотографиях образцов, приведенных на рис. 1.

На рис. 1 а светлые участки размерами 200-300 мкм, представляющие собой частицы полиэтилена, разделены четкими границами из углеродных нанотрубок. Такая структура получена благодаря тому, что при перемешивании порошка СВМПЭ с суспензией МУНТ, имеющей температуру $90{ }^{\circ} \mathrm{C}$, частицы полиэтилена набухают лишь у самой поверхности, поэтому наночастицы могут расположиться только в промежутках между ними. Нанотрубки взаимно перекрываются и контактируют друг с другом, образуя длинные проводящие цепочки, пронизывающие весь объем материала. Поэтому образец, приготовленный по данной методике, обладает повышенной проводимостью (рис. $2 a$ ).

В структуре образца № 2 (рис. 1б) наблюдаются скопления УНТ размерами от 10 до 200 мкм (черные участки), разделенные светлыми участками полиэтилена. Перемешивание СВМПЭ с суспензией МУНТ при температуре $110{ }^{\circ} \mathrm{C}$ в течение 10 мин приводит к набуханию части полимера. Так как набухание полиэтилена непосредственно связано с проникновением растворителя в пространство между полимерными цепями, то часть МУНТ, диспергированных в нем, также внедряется в разбухшие области. Таким образом, определенное количество МУНТ в дальнейшем оказывается изолированным полиэтиленом от основного массива нанотрубок, 
распределенных между частицами СВМПЭ. Поэтому проводимость образца № 2 (рис. $3 a$ ) ниже, чем у № 1.

Совершенно иная картина распределения нанотрубок зафиксирована в образце № 3 (рис. 1в). В нем МУНТ равномерно расположены по матрице в виде мельчайших агрегатов, подавляющее большинство которых имеет размеры не более 5-10 мкм. Агрегаты изолированы друг от друга полиэтиленом. Причиной этому является то, что в процессе ультразвуковой обработки смеси СВМПЭ/МУНТ в ксилоле с температурой $144{ }^{\circ} \mathrm{C}$ полиэтилен разбухает по всему объему, а нанотрубки равномерно распределяются в объёме матрицы, оказываясь изолированными друг от друга полимером. В этом случае в материале отсутствует сеть непрерывных контактов МУНТ, поэтому проводимость образца № 3 настолько низкая (рис. 4a), что его можно отнести к классу диэлектриков.

\section{Заключение}

Таким образом, метод смешивания СВМПЭ и МУНТ в растворителе позволяет получить как электропроводящие, так и диэлектрические композиты. Величина проводимости зависит не только от концентрации углеродных нанотрубок, но и от структуры их распределения в полимерной матрице, которая изменяется в соответствии с температурными условиями перемешивания компонентов композита.

Работа выполнена при поддержке Фонда содействия развитию малых форм предприятий в научно-технической сфере в рамках договора № 9594ГУ/2015 от 01.02.2016.

\section{Список литературы}

[1] Елецкий А.В., Книжник А.А., Потапкин Б.В., Кенни Х.В. Электрические характеристики полимерных композитов, содержащих углеродные нанотрубки, Успехи физических наук, 2015, 185(3), 225-270 [Eletskii A.V., Knizhnik A.A., Potapkin B.V., Kenny J.M. Electrical characteristics of carbon nanotube doped composites, Uspekhi Fizicheskikh Nauk, 2015, 185(3), 225-270 (in Russian)]

[2] Meschi Amoli B., Ahmad Ramazani S.A., Hadi Izadi. Preparation of Ultrahigh-MolecularWeight Polyethylene/Carbon Nanotube Nanocomposites with a Ziegler-Natta Catalytic System and Investigation of Their Thermal and Mechanical Properties, Journal of Applied Polymer Science, 2012, $125,453-461$.

[3] Lisunova M.O., Mamunya Ye.P., Lebovka N.I., Melezhyk A.V. Percolation behaviour of ultrahigh molecular weight polyethylene/multi-walled carbon nanotubes composites, European Polymer Journal, 2007, 43, 949-958.

[4] Kazakova M.A. Kuznetsov V.L., Semikolenova N.V., Moseenkov S.I., Krasnikov D.V., Matsko M.A., Ishchenko A.V., Zakharov V.A., Romanenko A.I., Anikeeva O.B., Tkachev E.N., Suslyaev V.I., Zhuravlev V.A., Dorozkin K.V. Comparative study of multiwalled carbon nanotube/ polyethylene composites produced via different techniques, Phys. Status Solidi B, 2014, 251(12), $2437-$ 2443.

[5] Mierczynska A., Mayne-L'Hermite M., Boiteux G., Jeszka J.K. Electrical and Mechanical Properties of Carbon Nanotube/Ultrahigh-Molecular-Weight Polyethylene Composites Prepared by a Filler Prelocalization Method, Journal of Applied Polymer Science, 2007, 105, 158-168. 
[6] Balogun Y.A., Buchanan R.C. Enhanced percolative properties from partial solubility dispersion of filler phase in conducting polymer composites (CPCs), Composites Science and Technology, 2010, 70, 892-900.

[7] Frohlich H. Theory of Dielectrics: Dielectric Constant and Dielectric Loss, Oxford, Clarendon Press, 1958, $200 \mathrm{p}$.

[8] Мотт, Н. Дэвис Э. Электронные процессы в некристаллических веществах. М.: Мир, 1982. T. 1, 260 c. [Mott N., Davis E. Electronic Processes in Non-Crystalline Materials, Moscow, Mir, 1982. V. 1, 260 p. (in Russian)]. 